\title{
Research Paper \\ Investigating the Anti-angiogenic Effects of Nanoemulsion Synthesized From Plant Anethum Graveolens L Essential Oil
}

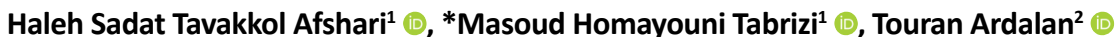

1. Department of Biology, Faculty of Basic Sciences, Mashhad Branch, Islamic Azad University, Mashhad, Iran. 2. Department of Chemistry, Faculty of Basic Sciences, Mashhad Branch, Islamic Azad University, Mashhad, Iran.

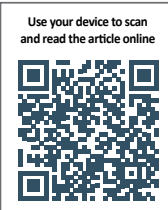

Citation: Tavakkol Afshari HS, Homayouni Tabrizi M, Ardalan T. [Investigation of Anti-angiogenic Effects of Nanoemulsion Synthesized From Plant Anethum Graveolens L Essential Oil (Persian)]. Journal of Arak University of Medical Sciences (JAMS). 2021; 24(1):62-73. https://doi.org/10.32598/JAMS.24.1.4042.8

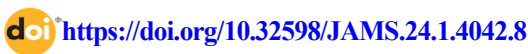

Keywords:

Angiogenesis, Chorioallantoic membrane, Anethum graveolens $\mathrm{L}$ essential nanoemulsion

\section{A B STRACT}

Article Info:

Received: 16 Feb 2020

Accepted: 24 Aug 2020 Available Online: 01 April 2021

\section{Extended Abstract}

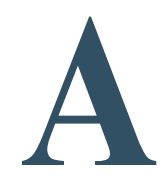

\section{Introduction}

ngiogenesis is necessary for organogenesis, proliferation, and cell differentiation in the embryonic period; it can be divided into two categories of physiology and pathology [1]. Angiogenesis plays an important role in the metastasis of cancerous tumors; thus, it can be targeted by antitumor therapies [4]. Studies indicated that the encapsulation and emulsification of vegetable oils due to the anti-cancer effects of these compounds can lead to their widespread use in inhibiting cancer cells. Studies have been conducted on the use of vegetable oils, like dill (Anethum graveolens L) [12] in the treatment

\section{* Corresponding Author:}

Masoud Homayouni Tabrizi, PhD.

Address: Department of Biology, Faculty of Basic Sciences, Mashhad Branch, Islamic Azad University, Mashhad, Iran

Tel: +98 (513) 8435000

E-mail: mhomayouni6@gmail.com 
of some cancers, including breast and liver types [11]. The present study examined the effects of different concentrations of dandelion essential oil nanoemulsion using the CAM method.

\section{Materials and Methods}

In preparing the nanoemulsion of the dill plant, Tween 20 and Tween 80, ethylene glycol, and distilled water were used. The nanoemulsion was prepared in the presence of ultrasonic waves or power of 200 watts for 60 minutes. In this study, 50 fertilized eggs of ROSS breed were divided into 5 groups, including control and laboratory control groups and 3 experimental groups; accordingly, they were placed in an incubator at $37^{\circ} \mathrm{C}$ and $65 \%$ humidity. On the second day of incubation, a window was made on the eggs. On day 8 of incubation, a gelatinous sponge (a combination of egg white albumin, isothermal agar solution, \& antibiotics) was placed on the chorioallantoic membrane. In the control group, $10 \mu \mathrm{L}$ of sterile distilled water was added to the gelatinous sponge on the chorioallantoic membrane; the laboratory control group was treated with $10 \mu \mathrm{L}$ of normal saline, followed by $10 \mu \mathrm{L}$ of different concentrations $(12.5,25, \& 50 \mu \mathrm{g} / \mathrm{mL})$ dill nanoemulsion, i.e., added to the sponge. On the $12^{\text {th }}$ day, the study samples were collected by stereomicroscope. J Image software was used to evaluate the length and number of vessels. Fetal height and weight were measured using calipers and scales, respec- tively. The obtained data were analyzed in SPSS by oneway Analysis of Variance (ANOVA) and Least Significant Difference (LSD) test.

\section{Results}

The morphological images of the chorioallantoic membrane in the study samples treated with different concentrations of nanoemulsion $(12.5,25, \& 50 \mu \mathrm{g} / \mathrm{mL})$, compared to the control and laboratory samples presented a decrease in the number of blood vessels (Figure 1). Exploring the number of blood vessels revealed no significant difference between the control and laboratory controls; however, there was a significant difference between the research samples treated with different concentrations of nanoemulsion and the controls ( $\left.{ }^{*} \mathrm{P}<0.05 \& * * * \mathrm{P}<0.001\right)$.

\section{Discussion and Conclusion}

Preventing the growth of cancer cells and inhibiting them can be performed by various strategies, such as inducing apoptosis or inhibiting angiogenesis. Thus, inhibiting the angiogenic process inhibits the growth and development of cancer cells. Recently, numerous anti-angiogenic drugs have been studied; however, most of them presented high toxicity and have developed drug resistance. Additionally, they failed to cross obstacles to achieve the desired goal [19]. Due to their small size, nanoemulsions can cross the
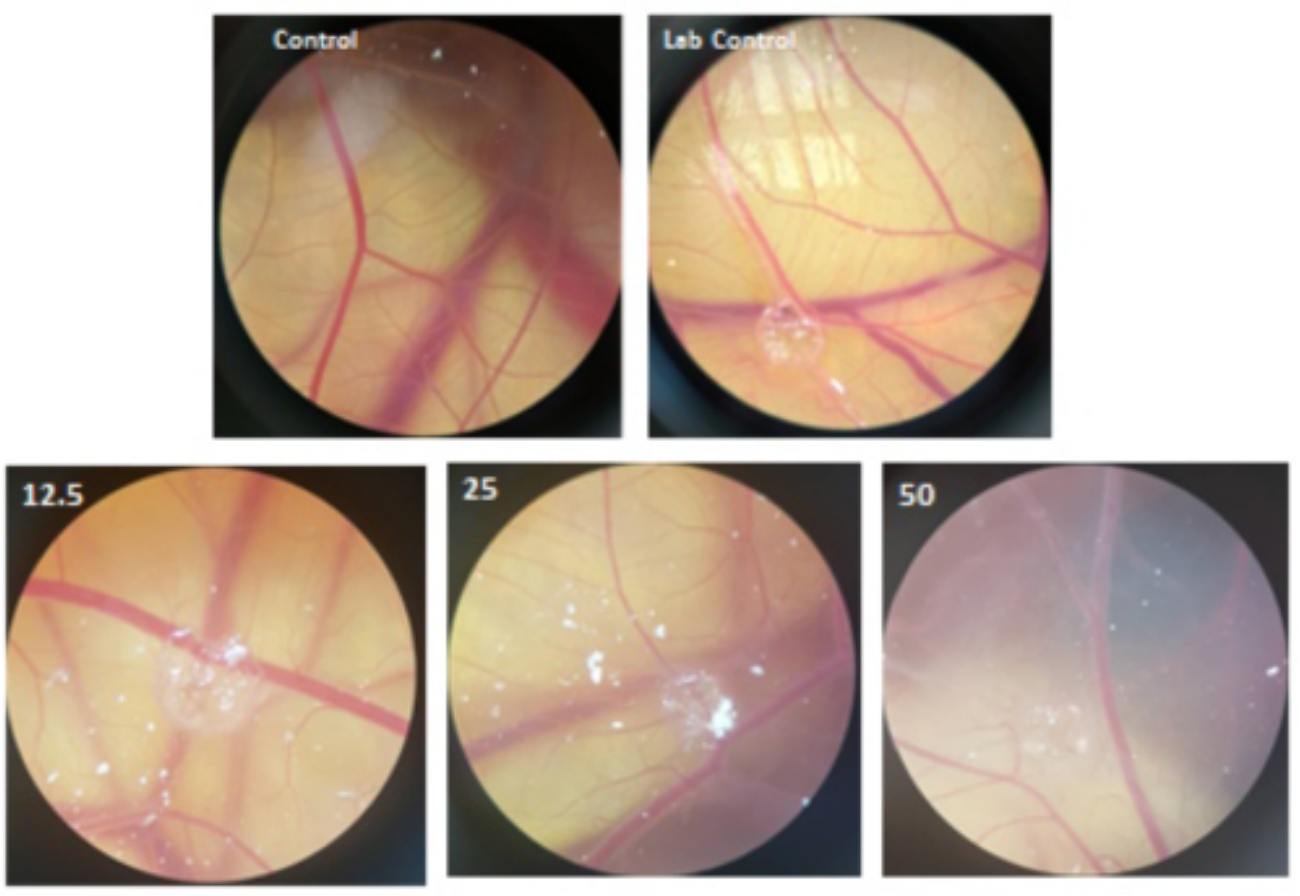

Figure 1. Photostreomicroscopic image of the chorioallantoic membrane of chick embryos in nano-emulsion-treated samples and control (without any treatment) and laboratory control (normal saline treatment) 
blood barrier and easily be present in tumor tissues; thus, they can exert their effects through the blood vessels surrounding the tumor. They can also be designed to encapsulate different drugs and select specific targets [20]. Dehelean et al. (2011) applied nanoemulsion with antiangiogenic properties for drug delivery. They investigated the antiangiogenic effect of nanoemulsion synthesized from flaxseed oil using the CAM method. The relevant results indicated that this combination of effects on vascular mesenchyme prevents angiogenesis [21]. Another study Dehelean et al. (2013) examined the effect of betulin nanoemulsion on angiogenesis in skin carcinoma cells in mice and chicken embryo chorion membranes. The related results also supported the anti-angiogenic effects of nanoemulsions [22]. Yousefian et al. (2020) explored the anti-angiogenic effect of nanoemulsion of lemon essential oil was using the CAM method; they concluded that this compound prevents angiogenesis [23]. In the present study, the anti-angiogenic effects of dill nanoemulsion were confirmed. This study, similar to the studies mentioned above, identified a significant reduction $(* * * \mathrm{P}<0.001)$ in the number and length of blood vessels in the chorioallantoic membrane of chickens.

\section{Ethical Considerations}

\section{Compliance with ethical guidelines}

All experiments of this research were carried out in compliance with ethical standards and according to the approval of the Research Ethics Committee of Mashhad Branch, Islamic Azad University (Code: IR.IAU.MSHD. REC.1398.027).

\section{Funding}

This research has not received any special funding from public, commercial or non-profit sector financing organizations.

\section{Authors' contributions}

Conceptualization: Masoud Homayouni Tabrizi Methodology: Masoud Homayouni Tabrizi, Turan Ardalan. Validation: Masoud Homayouni Tabrizi, Turan Ardalan. Analysis, Research: Haleh Sadat Tavakol Afshari. Draft: Haleh Sadat Tavakol Afshari. Edited and finalized by: Masoud Homayouni Tabrizi, Turan Ardalan. Visualization, Supervision, Project Management, Financing: Masoud Homayouni Tabrizi.

\section{Conflicts of interest}

The authors declared no conflicts of interest. 


\title{
بررسى اثرات ضدرَّزايى نانوامولسيون سنتزشده از اسانس كياه شويد
}

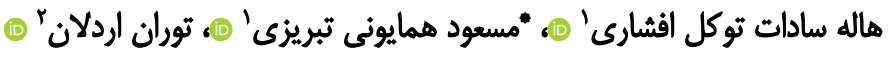 \\ ا. كروه زيست شناسى، نانشكده علوم، واحد مشهيد، دانشكاه آزاد اسلامى، مشهيه، ايران.

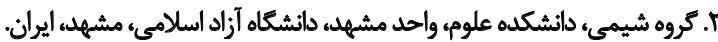

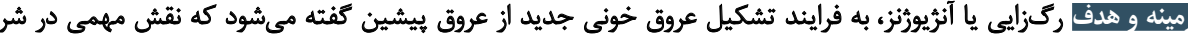

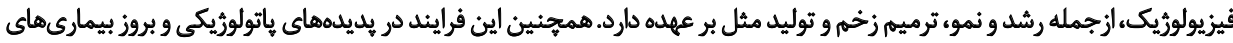

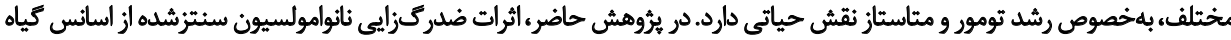

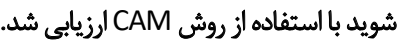

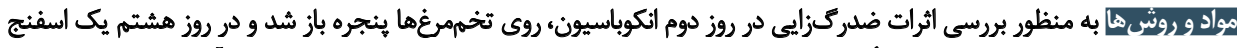

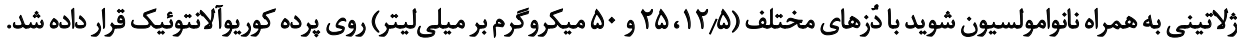

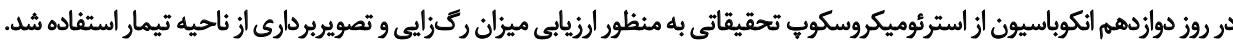

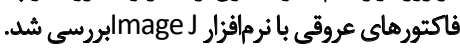

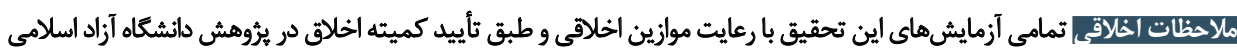
واحد مشهيد انجام كرفت (كد: IR.IAU.MSHD.REC.1398.027).

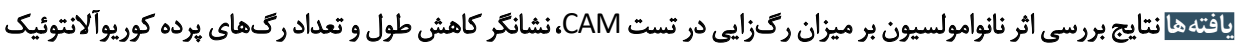

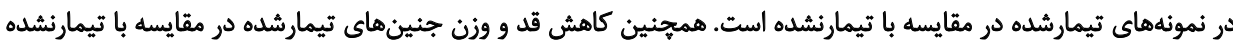

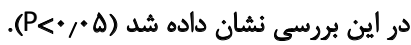

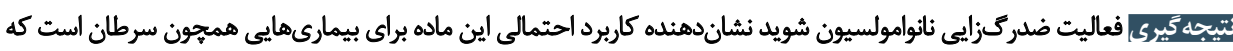

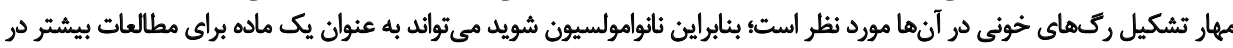

آنزيورثز فيزيولوزيك، فرايندى است به شدت تنظيمشده كه

مقدمه

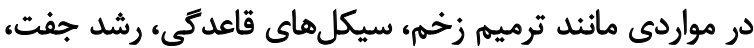

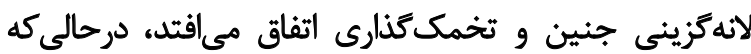

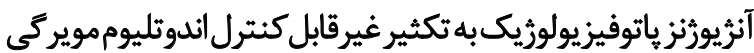

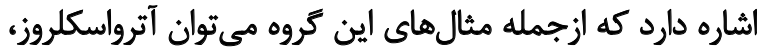

اندومتريوز و رتينوياتى ديابتى را نام برد [1ا].

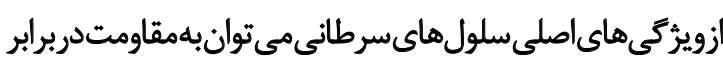

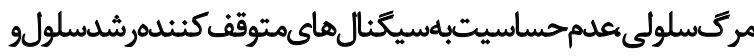

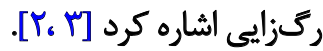
از آنجا كه آنثيوثنز در مثاستاز تومورهاى سرطاني داراي نقش رتش

آنزيوزنز به فرايند بيولوزيكى تشكيل ركهاى جديد از ركهاى

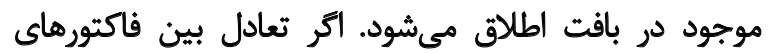

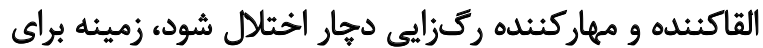

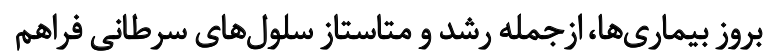

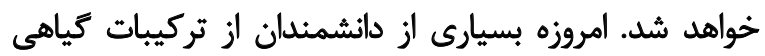

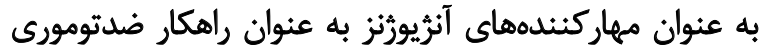

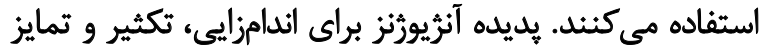

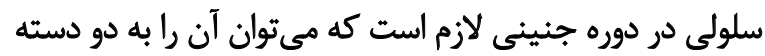

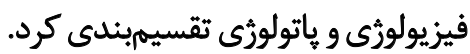

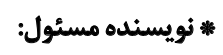

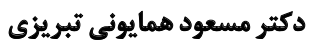

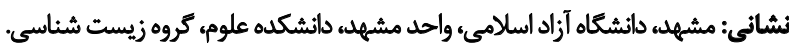

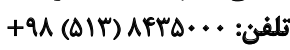

$$
\begin{aligned}
& \text { بهت الكترونيكي: mhomayouni6@gmail.com }
\end{aligned}
$$


در اين بررسى از نانوامولسيون تهيهشده از اسانس كياه شويد،

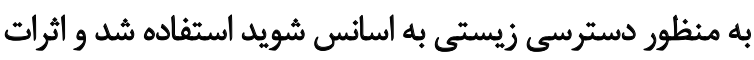
اين نانوامولسيون بر ميزان ركزايى با استفاده از روش

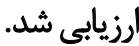

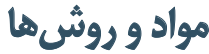

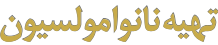

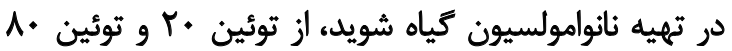

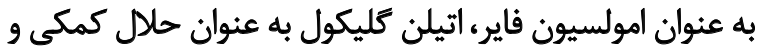

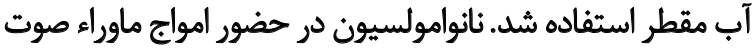

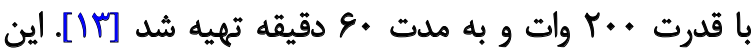

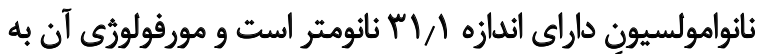
شكل تقريبا كروى گزارش شده است.

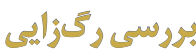

به منظور بررسى فعاليت آنثيورنز از تست CAM استفاده شد.

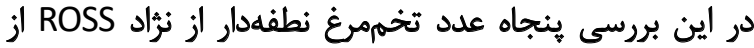

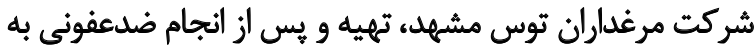

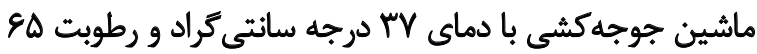

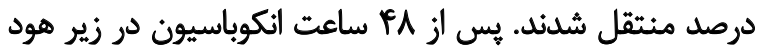

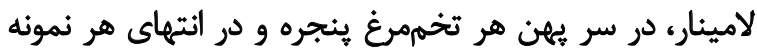

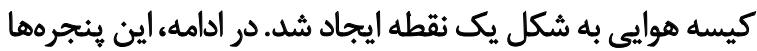

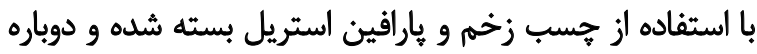
تخممغ بها به انكوباتور انتقال يافتند.

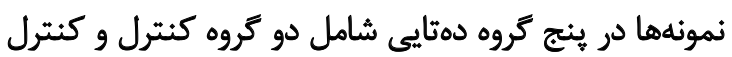

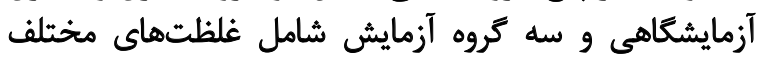

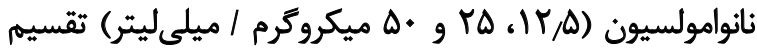

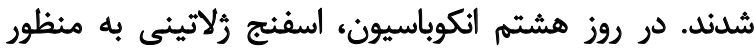

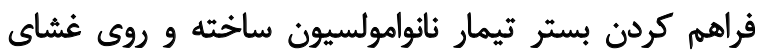

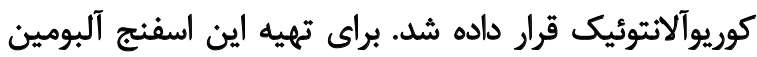

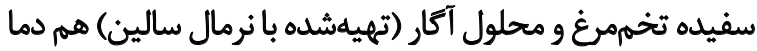

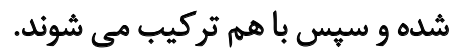

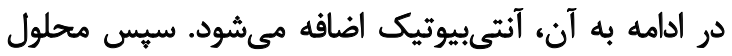

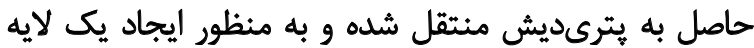

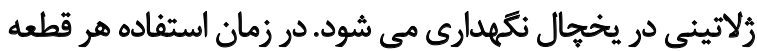

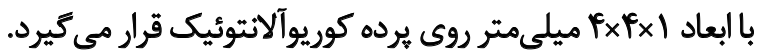
كروه آزمايش در شرايط طبيعى نكهيـدارى و بـهـ اسفنج

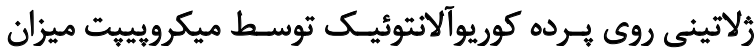

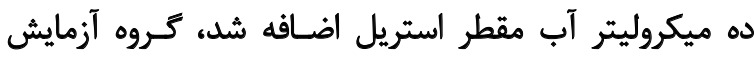

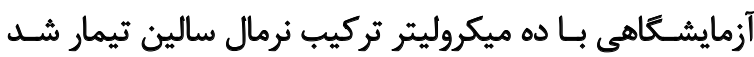

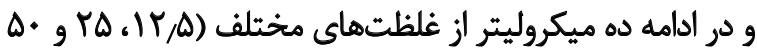
ميكروكرم بر ميلىليتر) نانوامولسيون شويد روى اسفنج اضافه
مهمى است، مىتواند هدف درمانهاى ضدتومورى قرار گيرد

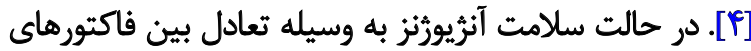

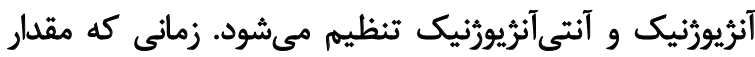

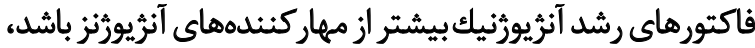

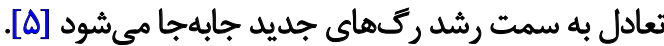

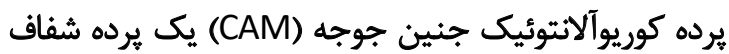
همراه با عروق خونى زياد است كه به به واسطه تركيب لائين لايههاى

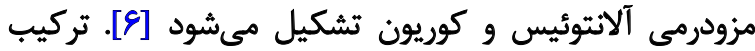

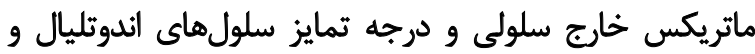

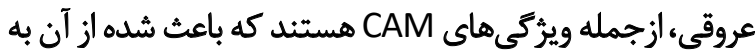

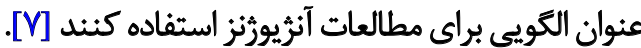
نانوامولسيون شبيه به ميكرو امولسيون است كه قطر ذرات

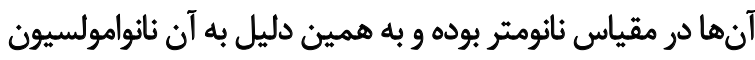

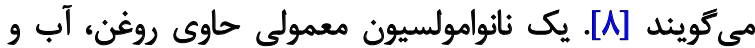
امولسيفاير (سورفاكتانت) است [ن]

سنتز نانوامولسيونها به روش هاي مختلف قابل انجام است كه

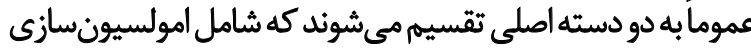

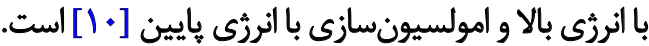

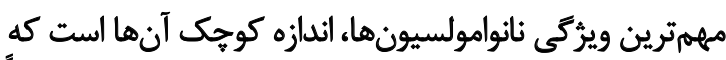

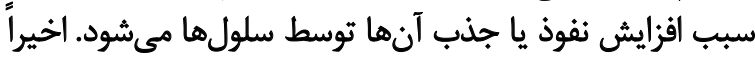
استفاده از نانوامولسيونها برائ مصارف دران درمان و دارو مورد توجه

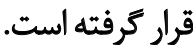

بررسىها نشان داده كه كيسوله كردن و امولسيفيكاسيون

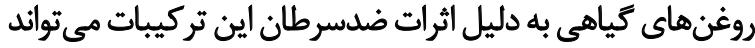

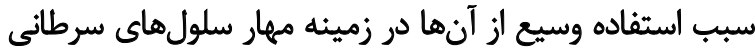

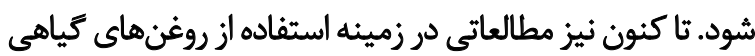

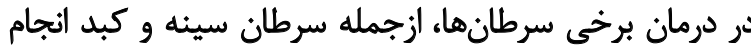

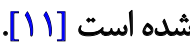

كياه دارويى شويد يكى از بهترين كياهان دارويى قابل تنذئ باديه

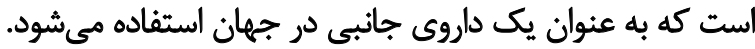

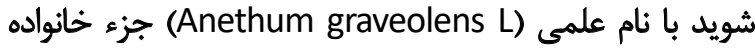

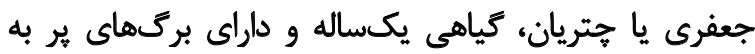

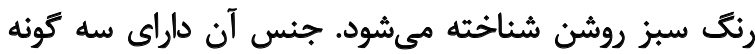

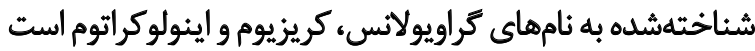

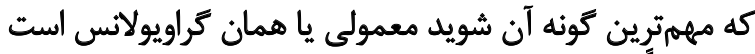

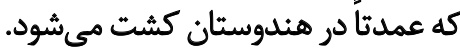

آنتريوم كراويولانس (شويد) حاوى موادى همجيون عصاره

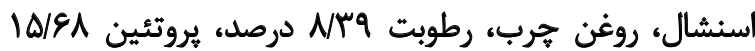

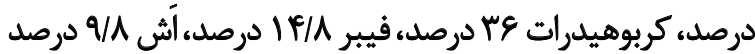

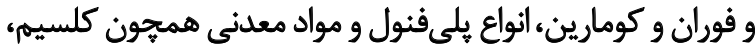

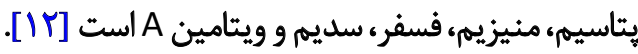




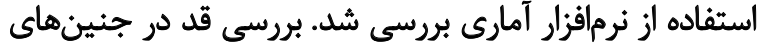

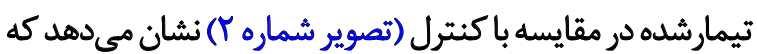

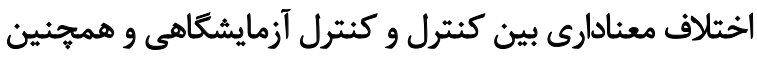

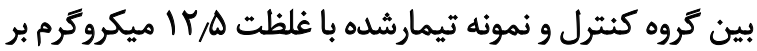

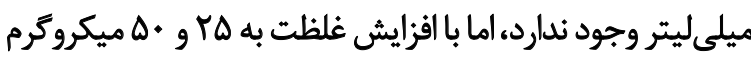

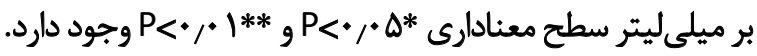
در ارزيابى وزن جنينها (تصوير شماره بآ) نيز هيج تفاوت

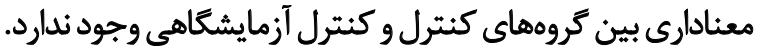

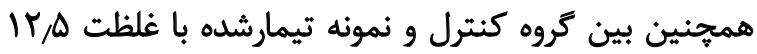

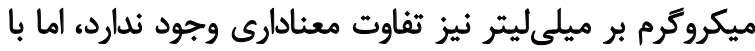

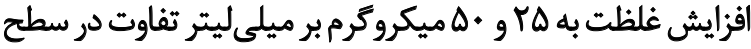

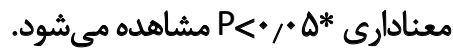

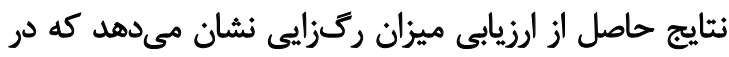

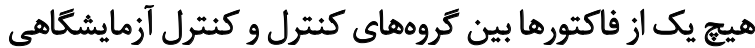

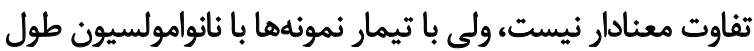

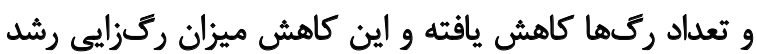
جنين (وزن و قد) را نيز تحت تأثير قرار مى دهد.

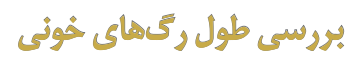

ارزيابى طول عروق خونى در كروههاى مختلف نشان ميدهد

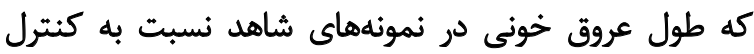

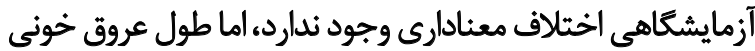

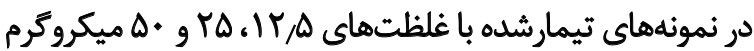

شد. يس از تيمار نمونههاء ينجره تخممرغها با باستفاده از جسب

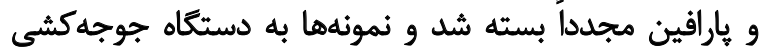

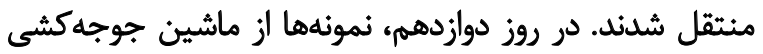

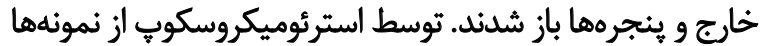
در محدوده اسفنج عكسبردارى صورت ترفت.

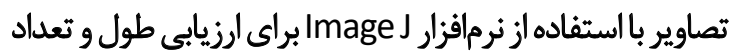

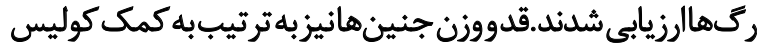

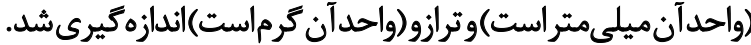

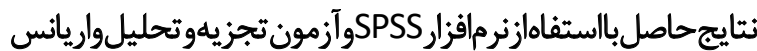
يكسرفه و آزمون LSD تحليل شدند.

يافتها

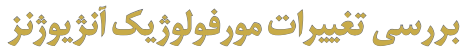

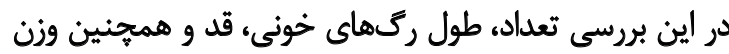

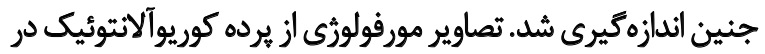

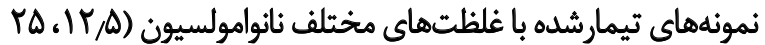

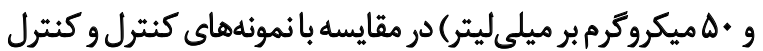

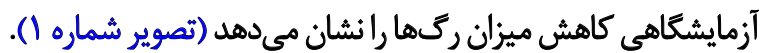

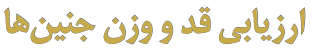

در اين مطالعه، وزن و قد جنينها با استفاده از ترازو و كوليس

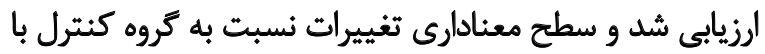
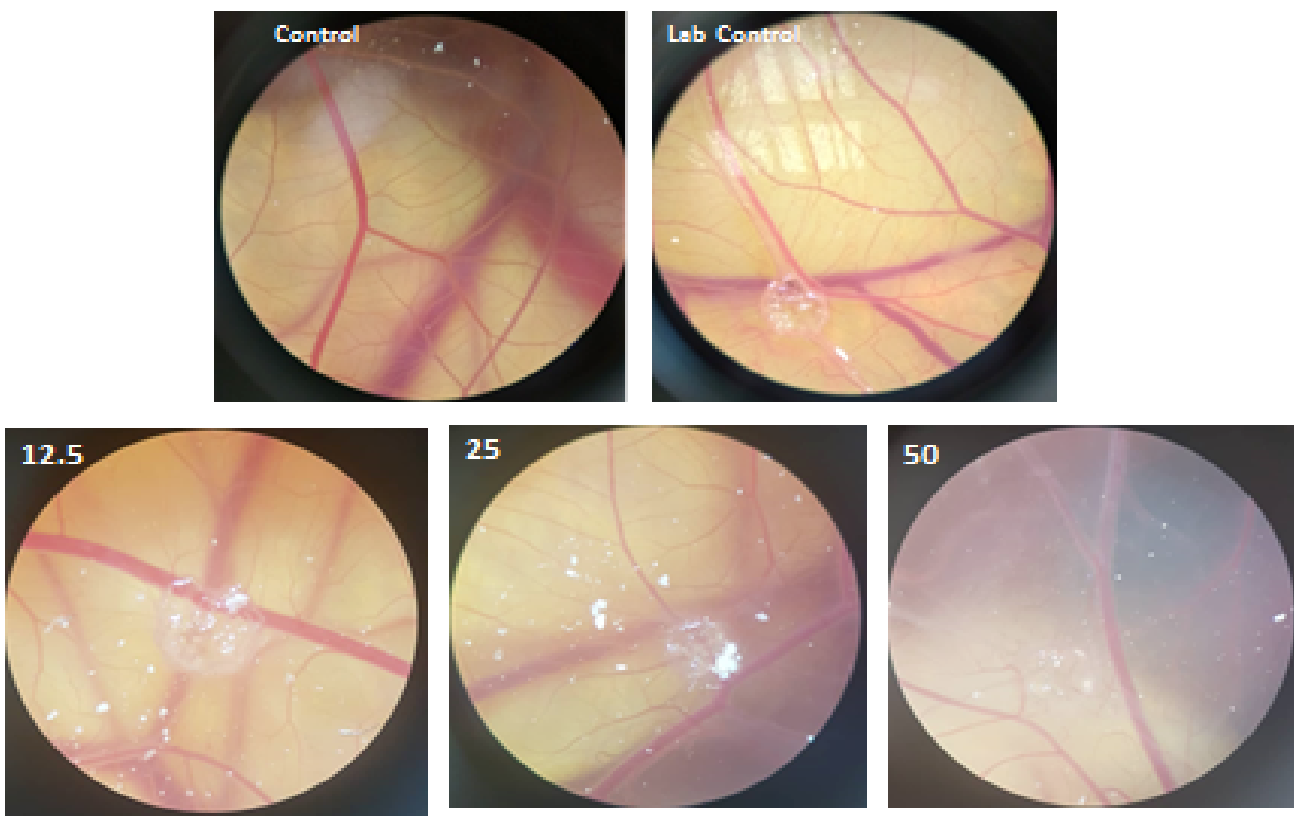

تصوير ا. تصوير استريوميكروسكوب از برده كوريوآلانتوئيك جنين جوجه در نمونههاي تيمار با نانوامولسيون اسائس شويد در مثايسه با تروهاي آزمايش و آزمايش آزمايشكاهي آتصوير 


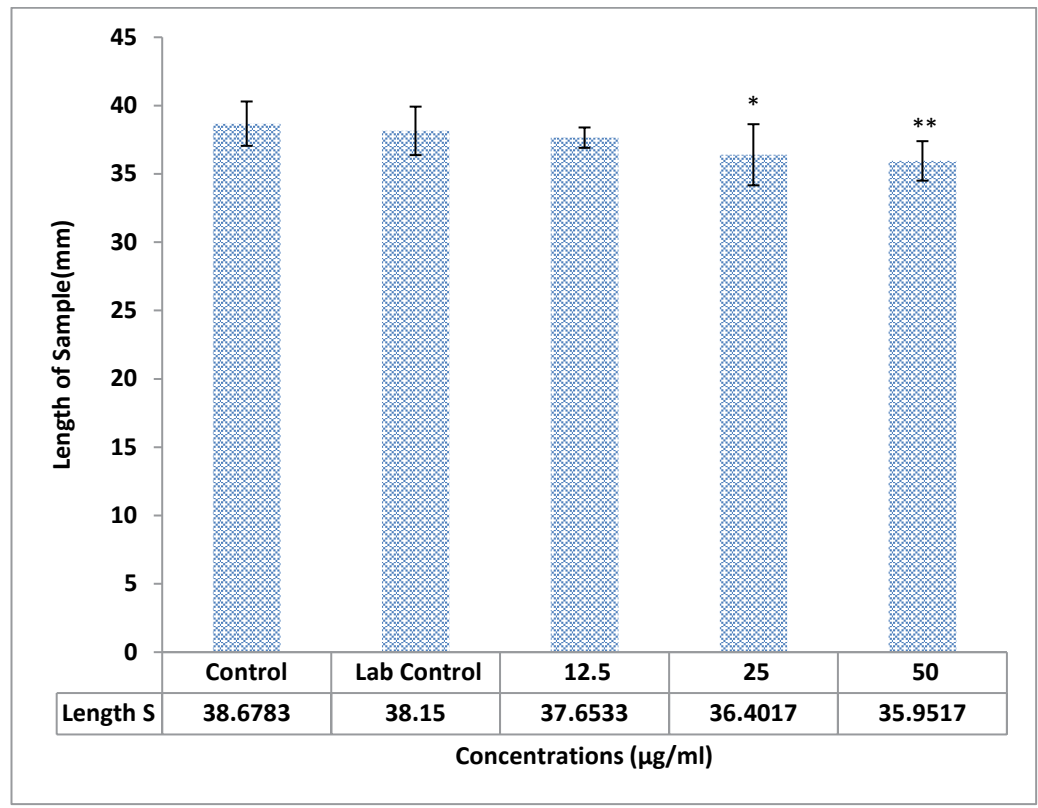

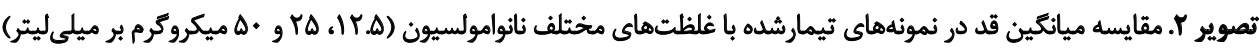

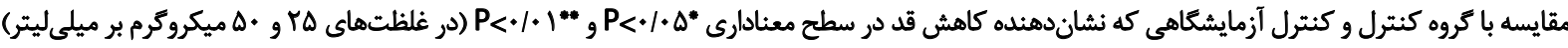

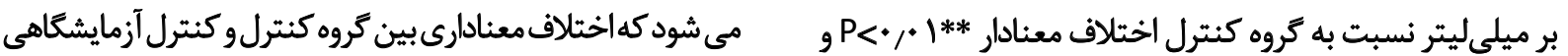

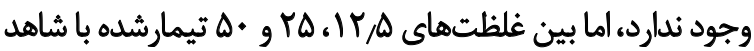

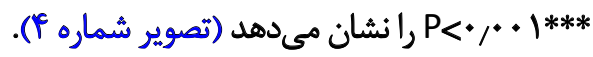

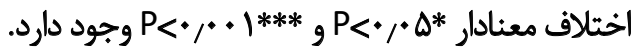
در بررسى تصوير شماره ه مربوط به تعداد عروق خونى مشاهده

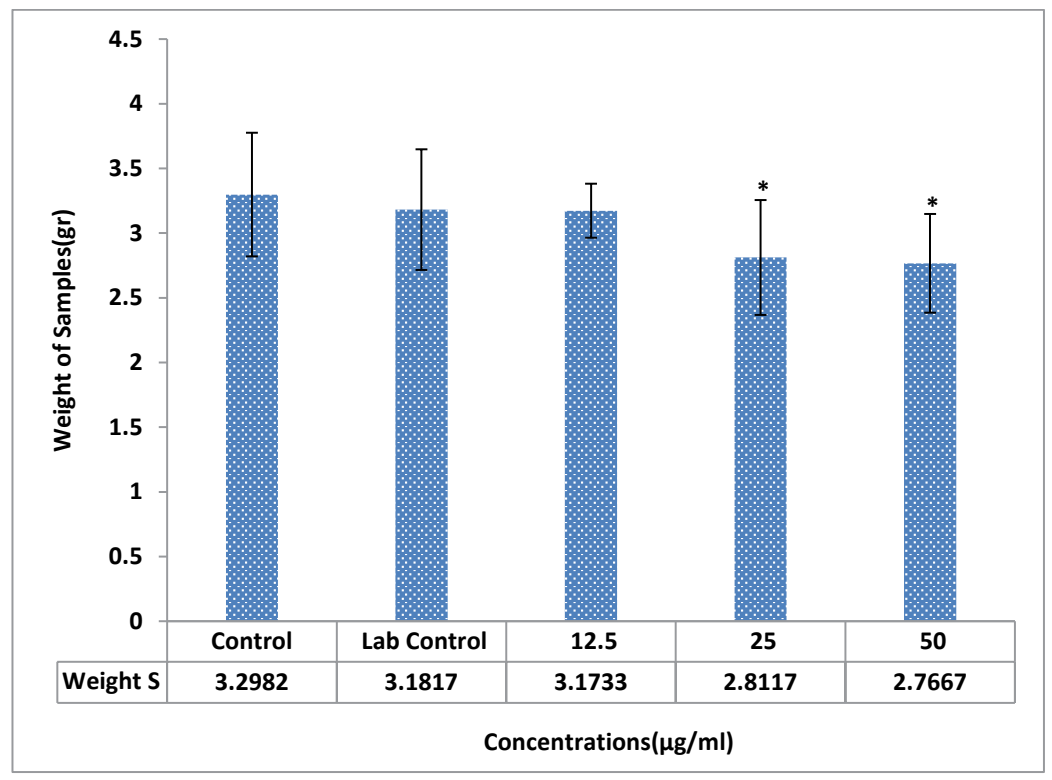

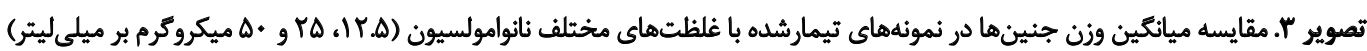

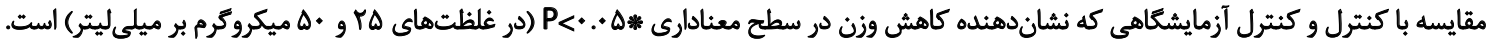




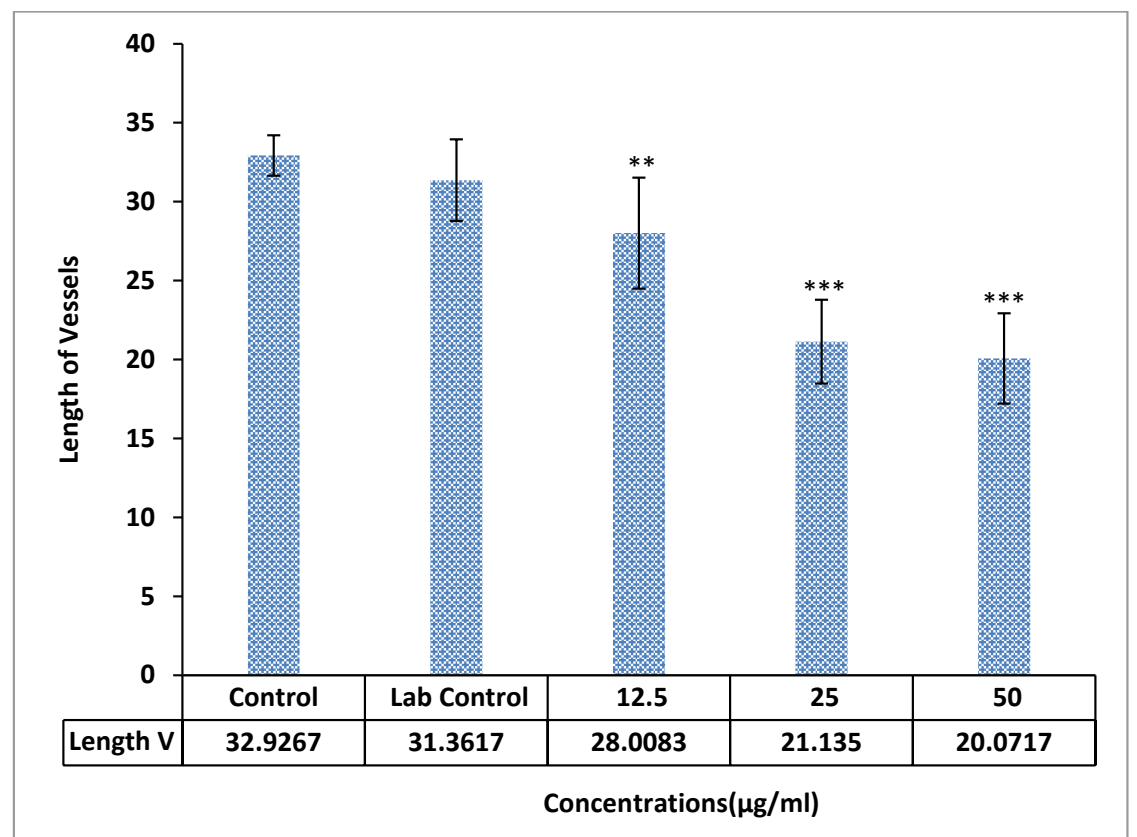

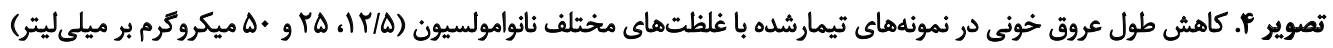

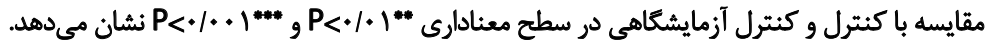

سيستم هايايزوتروبيك ثابتى هستند وكاربردهاى زيادى دارند [ [ I F].

ث

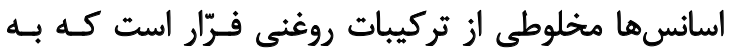

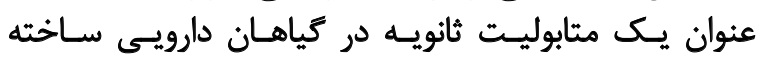

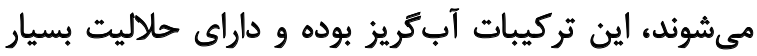

نانوامولسيونها، امولسيونهاى بسيار ريزشده يا امولسيونهاى إناى

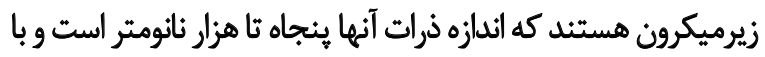

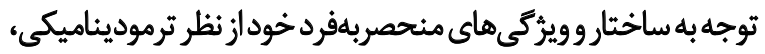

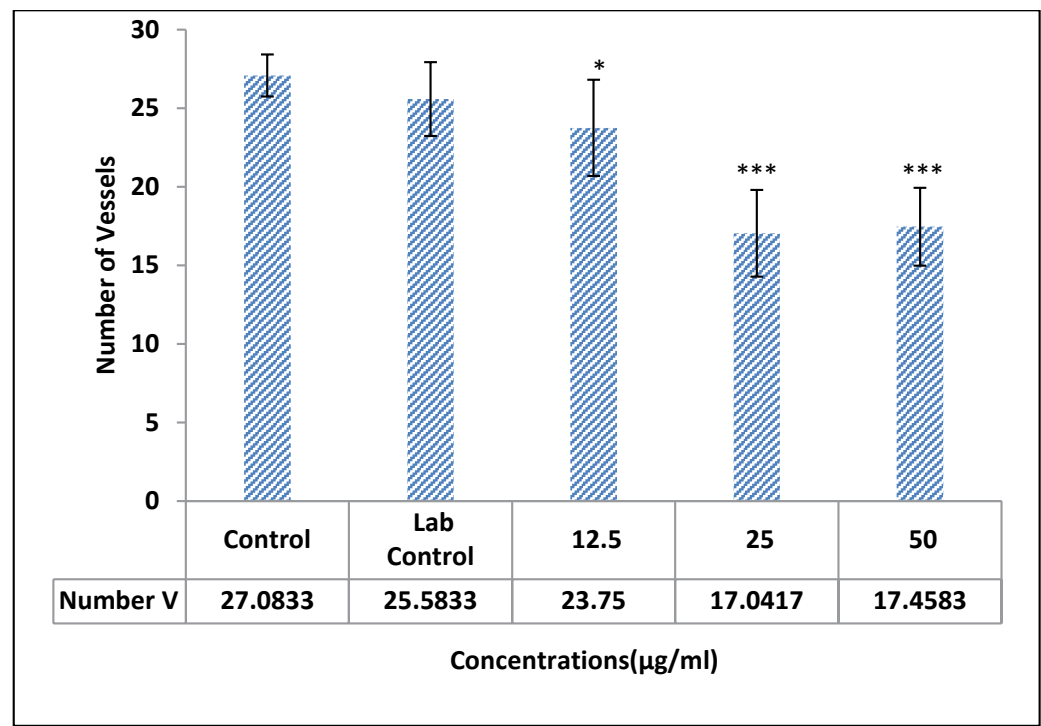

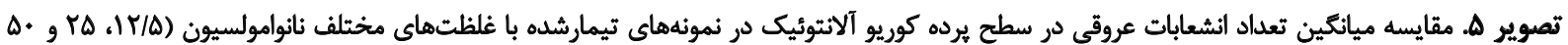
ميكروكرم بر ميلى مقايسه ميانين

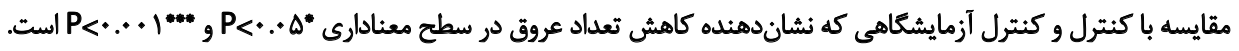


نانومولسيونها با توجه به اندازه كوجك خود توانايي عبور از

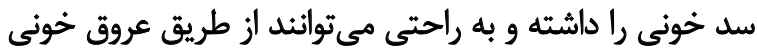

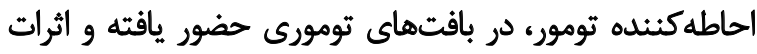

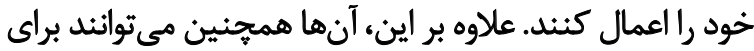

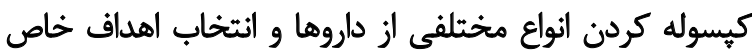

طراحى شوند [r·r].

در ئروهش دهلين' و همكاران از نانوامولسيون داراى

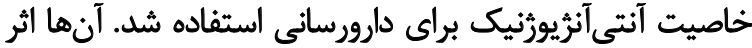

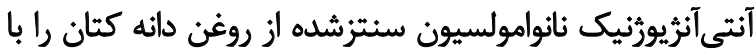

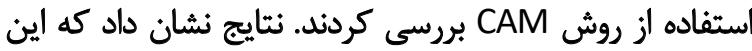

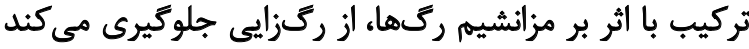

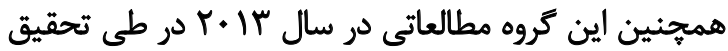

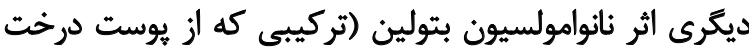

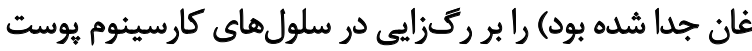

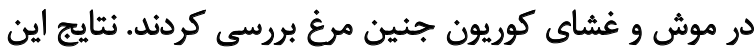

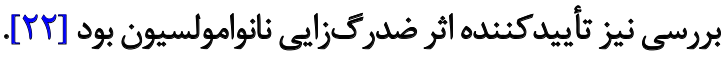
درمطالعهيوسفيانوهمكاراناثرآنتئاترئيورثيكنانواموامولسيوناسانس

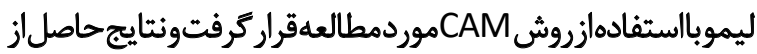

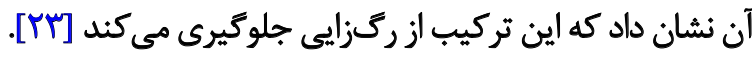
در مطالعه حاضر اثرات ضدركزايي نانوامولسيون كياه شويد

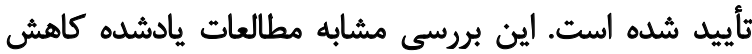

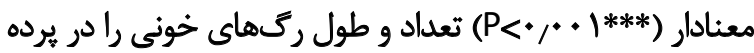
كوريوآلانتوئيك جوجه نشان داد.

تثيجه نَّيرى

در يروهش حاضر، اثرات ركزايى نانوامولسيون سنتزشده از

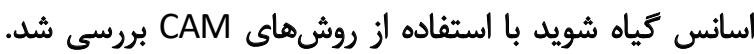

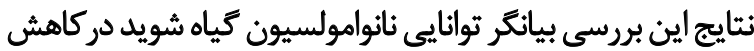

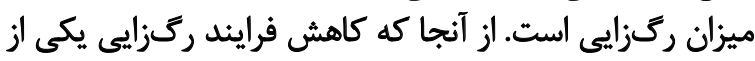

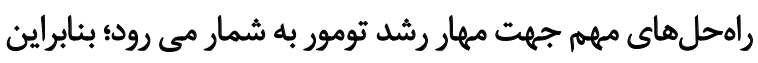

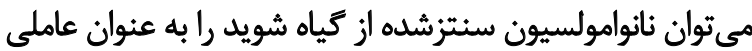

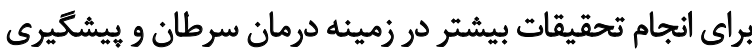

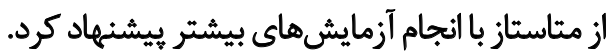

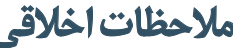

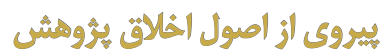

تمامى آزمايشهاى اين تحقيق بارعايت موازين اخلاقى وطبق

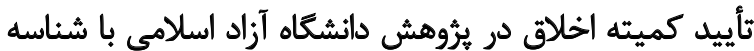
مصوبه IR.IAU.MSHD.REC.1398.027 انجام شد.
يايينى در آب هستند و به راحتى توسط كرما، نور و هوا اكسيده

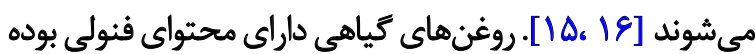

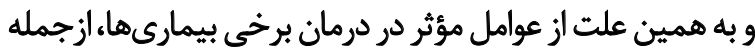

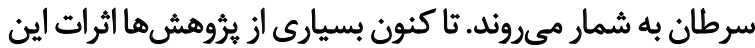

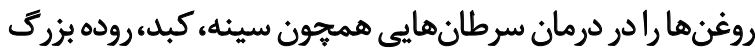
و... بروسى كرداند.

مههمترين مشكل در بهكاركيرى اين تركيبات حلاليت ضعيف

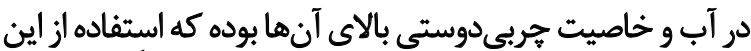

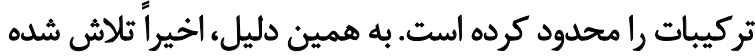

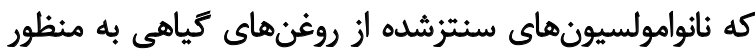
افزايش سازكارى زيستى و اثربخشى بيشتر مورد استفاده قرار

كيرد [11].

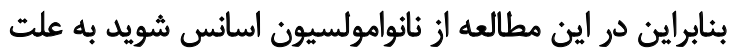

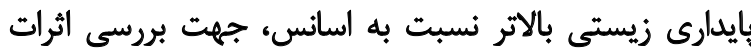

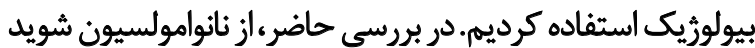

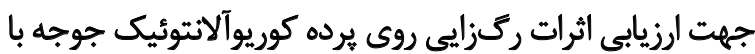

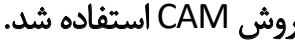

نتايج حاصل از بررسى ميزان ركزائى نشان ميدهد كه

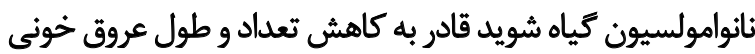

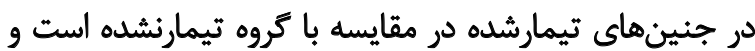
ممكن است كاهش رگز اييى سبب كاهش رش رشد جنين (كاهش

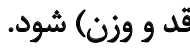

جلوكيرى از توسعه سلول هاي سرطانى و مهار آنها مي تواند با

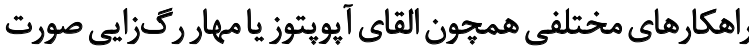

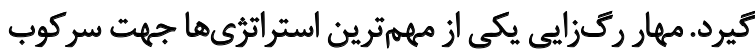

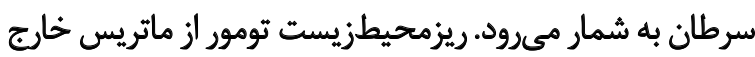

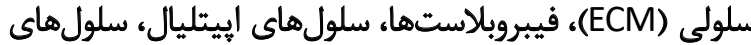

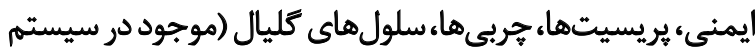

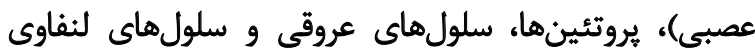
تشكيل شده است. ت بروتين.

آنزيوزنز جزء مهمى از متاستاز تومور است و مى تواند متاستاز

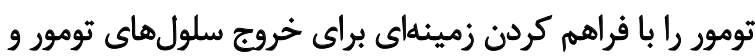

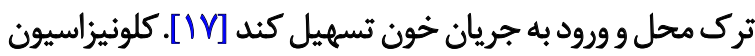

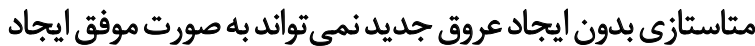

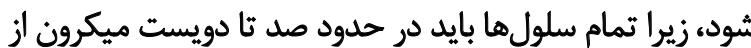

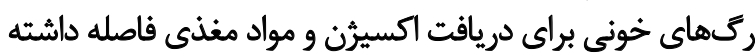

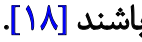

بنابراين مهار فرايند رگزايى، سبب مهار رشد و توسعه

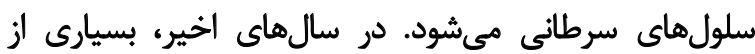

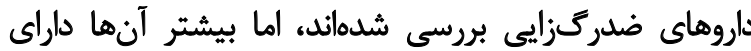

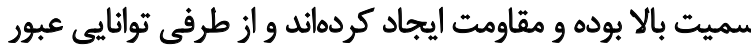
از موانع براي رسيدن به هدف مورد نظر را ندارند [19]. 


$$
\text { Ithe collo }
$$

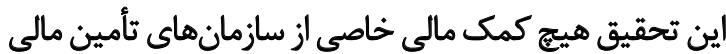

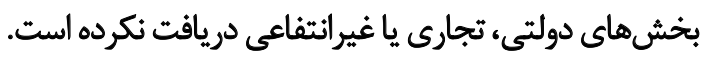

\section{il}

مفهومسازى: مسعود همايونى تبريزى؛ روششئاسى: مسعود

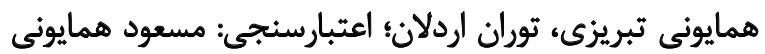

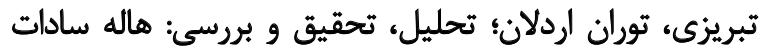

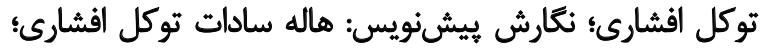

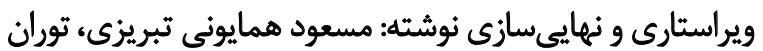

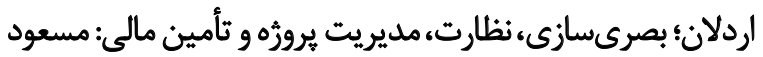

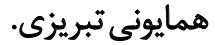

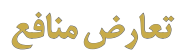
طبق نظر نويسندكان اين مقاله هيج كونه تعارض منافعى ندارد. 


\section{Reference}

[1] Van RN, Piek JJ, Schaper W, Bode C, Buschmann I. Arteriogenesis: Mechanisms and modulation of collateral artery development. J Nucl Cardiol. 2001; 8(6):687-93. [DOI:10.1067/mnc.2001.118924] [PMID]

[2] Korir A, Mauti N, Moats P, Gurka MJ, Mutuma G, Metheny C, et al. Developing clinical strength-of-evidence approach to define HIV-associated malignancies for cancer registration in Kenya. PloS one. 2014; 9(1):e85881. [DOI:10.1371/journal.pone.0085881] [PMID] [PMCID]

[3] Fiaschi T, Chiarugi P. Oxidative stress, tumor microenvironment, and metabolic reprogramming: A diabolic liaison. Int J Cell Biol. 2012; 2012:762825. [DOI:10.1155/2012/762825] [PMID] [PMCID]

[4] Kouhestanian K, Baharara J, Ramezani T, Mousavi M. [Antiangiogenic effects of eugenol inchorioallantoic membrane of chick embryo (Persian)]. J Shahid Sadoughi Univ Med Sci. 2015; 23(4):2109-17. https:// jssu.ssu.ac.ir/browse.php?a_code=A-10-1624-2\&slc_lang=en\&sid=1

[5] Salehi E, Amjadi FS, Khazaei M. [Angiogenesis in health and dDisease: Role of Vascular Endothelial Growth Factor (VEGF) (Persian)]. J Isfahan Med Sch. 2011; 29(132):312-26. https://www.sid.ir/fa/Journal/ViewPaper.aspx?ID=139629

[6] Attarian F, Zafar Bala Nezhad S, Tehrani pour M. [The effect of stachys lavandulifolia alcoholic extract on angiogenesis in chorioallantoic membrane of ross chicken embryo (Persian)]. J Shahid Sadoughi Univ Med Sci. 2016; 23(11):1084-93. https://jssu.ssu.ac.ir/browse.php?a_code=A10-2257-1\&slc_lang=en\&sid=1

[7] Ribatti D. The chick embryo chorioallantoic membrane as an in vivo assay to study antiangiogenesis. Pharmaceuticals. 2010; 3(3):482-513. [DOI:10.3390/ph3030482] [PMID] [PMCID]

[8] Gutiérrez JM, González C, Maestro A, Solè IM, Pey CM, Nolla J. Nanoemulsions: New applications and optimization of their preparation. Curr Opin Colloid Interface Sci. 2008; 13(4):245-51. [DOI:10.1016/j.cocis.2008.01.005]

[9] Gupta A, Eral HB, Hatton TA, Doyle PS. Nanoemulsions: Formation, properties and applications. Soft Matter. 2016; 12(11):2826-41. [DOI:10.1039/C5SM02958A] [PMID]

[10] Tadros T, Izquierdo P, Esquena J, Solans C. Formation and stability of nano-emulsions. Adv Colloid Interface Sci. 2004; 108-9:303-18. [DOI:10.1016/j.cis.2003.10.023] [PMID]

[11] Nirmala MJ, Nagarajan R. Microemulsions as potent drug delivery systems. J Nanomed Nanotechnol. 2016; 7:e139. [DOI:10.4172/21577439.1000e139]

[12] Mohammed GJ, Kadhim MJ, Hussein HM. Characterization of bioactive chemical compounds from Aspergillus terreus and evaluation of antibacterial and antifungal activity. Int J Pharmacogn Phytochem Res. 2016; 8(6):889-905. https://www.researchgate.net/profile/MohanadJawad/publication/304945402_Characterization_of_bioactive_chemical_compounds_from_aspergillus_terreus_and_evaluation_of_antibacterial_and_antifungal_activity/links/57e2482908ae9e25307eb3ba/ Characterization-of-bioactive-chemical-compounds-from-aspergillusterreus-and-evaluation-of-antibacterial-and-antifungal-activity.pdf

[13] Tavakkol Afshari HS, Homayouni Tabrizi M, Ardalan T. [Evaluation of Antioxidant and Anticancer Effects of Nanoemulsions Prepared Using Dill Essential Oil (Persian)]. J Arak Univ of Med Sci. 2019; 22(4):40-51. [DOI:10.32598/JAMS.22.4.40]

[14] Jaiswal M, Dudhe R, Sharma PK. Nanoemulsion: An advanced mode of drug delivery system. 3 Biotech. 2015; 5(2):123-7. [DOI:10.1007/ s13205-014-0214-0] [PMID] [PMCID]
[15] Geng S, Cui Z, Huang X, Chen Y, Xu D, Xiong P. Variations in essential oil yield and composition during Cinnamomum cassia bark growth. Ind Crops Prod. 2011; 33(1):248-52. [DOI:10.1016/j.indcrop.2010.10.018]

[16] Sabzghabaee AM, Nili F, Ghannadi A, Eizadi-Mood N, Anvari M. Role of menthol in treatment of candidial napkin dermatitis. World J Pediatr. 2011; 7(2):167-70. [DOI:10.1007/s12519-011-0253-0] [PMID]

[17] Mäkelä S. [Use of mesoporous torture particles as a drug carrier in the development of therapies for visceral leishmaniasis (Finnish)] [MSc. Thesis]. Kuopio, Finland: University of Eastern Finland. 2015. https://erepo. ueffi/bitstream/handle/123456789/16201/urn_nbn_fi_uef-20151124. pdf?sequence $=-1$

[18] Paduch R. The role of lymphangiogenesis and angiogenesis in tumor metastasis. Cell Oncol. 2016; 39(5):397-410. [DOI:10.1007/s13402-0160281-9] [PMID] [PMCID]

[19] Tan W, Wang H, Chen Y, Zhang X, Zhu H, Yang C, et al. Molecula aptamers for drug delivery. Trends Biotechnol. 2011; 29(12):634-40. [DOI:10.1016/j.tibtech.2011.06.009] [PMID] [PMCID]

[20] Sandip T, Yi-Meng T, Mansoor A. Preparation and in vitro characterization of multifunctional nanoemulsions for simultaneous MR imaging and targeted drug dleivery. J Biomed Nanotechnol. 2006; 2(3-4):217-24. [DOI:10.1166/jbn.2006.038]

[21] Dehelean CA, Feflea S, Ganta S, Amiji M. Anti-angiogenic effects of betulinic acid administered in nanoemulsion formulation using chorioallantoic membrane assay. J Biomed Nanotechnol. 2011; 7(2):317-24. [DOI:10.1166/jbn.2011.1297] [PMID]

[22] Dehelean CA, Feflea S, Gheorgheosu D, Ganta S, Cimpean AM, Muntean $D$, et al. Anti-angiogenic and anti-cancer evaluation of betulin nanoemulsion in chicken chorioallantoic membrane and skin carcinoma in Balb/c mice. J Biomed Nanotechnol. 2013; 9(4):577-89. [DOI:10.1166/ jbn.2013.1563] [PMID]

[23] Yousefian Rad E, Homayouni Tabrizi M, Ardalan P, Seyedi SM, Yadamani S, Zamani-Esmati $P$, et al. Citrus Lemon Essential Oil Nanoemulsion (CLEO-NE), a safe cell-depended apoptosis inducer in human A549 lung cancer cells with anti-angiogenic activity. J Microencapsul. 2020; 37(5):394-402. [DOI:10.1080/02652048.2020.1767223] [PMID] 
This Page Intentionally Left Blank 